\title{
Distinct inflammatory and cytopathic characteristics of Escherichia coli isolates from inflammatory bowel disease patients
}

Jensen, Stina Rikke; Mirsepasi-Lauridsen, Hengameh Chloé; Thysen, Anna Hammerich; Brynskov, Jørn; Krogfelt, Karen Angeliki; Petersen, Andreas Munk; Pedersen, Anders Elm; Brix, Susanne

\section{Published in:}

International Journal of Medical Microbiology

Link to article, DOI:

10.1016/j.ijmm.2015.10.002

Publication date:

2015

Document Version

Peer reviewed version

Link back to DTU Orbit

Citation $(A P A)$ :

Jensen, S. R., Mirsepasi-Lauridsen, H. C., Thysen, A. H., Brynskov, J., Krogfelt, K. A., Petersen, A. M., Pedersen, A. E., \& Brix, S. (2015). Distinct inflammatory and cytopathic characteristics of Escherichia coli isolates from inflammatory bowel disease patients. International Journal of Medical Microbiology, 305(8), 925936. https://doi.org/10.1016/j.jimm.2015.10.002

\section{General rights}

Copyright and moral rights for the publications made accessible in the public portal are retained by the authors and/or other copyright owners and it is a condition of accessing publications that users recognise and abide by the legal requirements associated with these rights.

- Users may download and print one copy of any publication from the public portal for the purpose of private study or research.

- You may not further distribute the material or use it for any profit-making activity or commercial gain

- You may freely distribute the URL identifying the publication in the public portal 


\section{Distinct inflammatory and cytopathic characteristics of Escherichia coli isolates from Inflammatory Bowel Disease patients}

Stina Rikke Jensen ${ }^{\mathrm{a}, 1}$, Hengameh Mirsepasi ${ }^{\mathrm{b}}$, Anna Hammerich Thysen ${ }^{\mathrm{a}}$, Jørn Brynskov ${ }^{\mathrm{c}}$, Karen A. Krogfelt $^{b}$, Andreas Munk Petersen ${ }^{\mathrm{b}, \mathrm{d}}$, Anders Elm Pedersen ${ }^{\mathrm{e}}$ and Susanne Brix ${ }^{\mathrm{a}, \#}$.

${ }^{a}$ Center for Biological Sequence Analysis, Dept. of Systems Biology, Technical University of Denmark,

${ }^{b}$ Dept. of Microbiology and Infection Control, Statens Serum Institut, Denmark, ${ }^{\mathrm{c} D e p t . ~ o f ~}$ Gastroenterology, Medical Section, Herlev Hospital, University of Copenhagen, Denmark, ${ }^{d}$ Dept. of Gastroenterology, Medical Section, Hvidovre Hospital, University of Copenhagen, Denmark, ${ }^{\mathrm{e} D e p t . ~ o f ~}$ International Health, Immunology and Microbiology, University of Copenhagen, Denmark.

\#Corresponding author: Susanne Brix, Center for Biological Sequence Analysis, Department of Systems Biology, Technical University of Denmark, sbp@bio.dtu.dk, Phone +45 21604264 .

${ }^{1}$ Present address: Insulin Biology, Novo Nordisk A/S, Copenhagen, Denmark 


\section{Abstract}

Escherichia coli (E. coli) may be implicated in the pathogenesis of inflammatory bowel disease (IBD), as implied from a higher prevalence of mucosa-associated $E$. coli in the gut of IBD-affected individuals. However, it is unclear whether different non-diarrheagenic E. coli spp segregate from each other in their ability to promote intestinal inflammation. Herein we compared the inflammation-inducing properties of non-diarrheagenic LF82, 691-04A, E. coli Nissle 1917 (ECN) and eleven new intestinal isolates from different locations in five IBD patients and one healthy control.

Viable E. coli were cultured with human monocyte-derived dendritic cells (moDCs) and monolayers of intestinal epithelial cells (IECS), followed by analysis of secreted cytokines, intracellular levels of reactive oxygen species and cellular death.

The IBD-associated E. coli LF82 induced the same dose-dependent inflammatory cytokine profile as

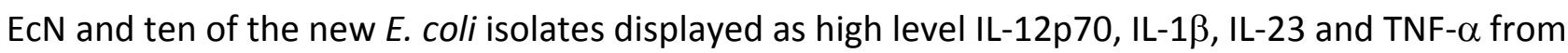
moDCs irrespective of their site of isolation (ileum/ colon/ faeces), disease origin (diseased/nondiseased) or known virulence factors. Contrarily, 691-04A and one new IBD E. coli isolate induced a different cellular phenotype with enhanced killing of moDCs and IECs, coupled to elevated IL-18.

The cytopathic nature of 691-04A and one other IBD E. coli isolate suggests that colonization with specific non-diarrheagenic E. coli could promote intestinal barrier leakage and profound intestinal inflammation, while LF82, ECN and the remaining non-diarrheagenic E. coli isolates hold notorious pro-inflammatory characteristics that can progress inflammation in case of intestinal barrier leakage. 
Key Words: IBD-associated E. coli, dendritic cells, inflammation, cytokine profile, cell death.

\begin{abstract}
Abbreviations:
CD: Crohn's disease, UC: Ulcerative colitis, IBD: Inflammatory bowel diseases, AIEC: Adherent-invasive

E. coli, DC: Dendritic cell, moDC: monocyte-derived DC, IEC: Intestinal epithelial cell, ECN: E. coli Nissle 1917, LPS: Lipopolysaccharide, MOI: Multiplicity of infection, ROS: Reactive oxygen species.
\end{abstract}




\section{Introduction}

Crohn's disease (CD) and ulcerative colitis (UC) are chronic inflammatory bowel diseases (IBD) believed to arise from defects in the gut barrier, combined with excessive immune responses to commensal gut microbes. Based on genome-wide association studies, genes involved in processing of invasive bacteria have been linked to $C D$, whereas genes associated with barrier function has primarily been linked to UC (Lees et al., 2011). Despite the fact that CD and UC are obviously distinct disorders, they share clinical and pathological features that appear to involve $E$. coli as increased numbers of $E$. coli have been identified in the gut mucosa of $C D$ and $U C$ patients compared to healthy controls (Kotlowski et al., 2007; Mondot et al., 2011; Sokol et al., 2006). Some of these IBD-associated E. coli isolates display strong host-interacting properties that include the ability to adhere to and invade the gut epithelium (Barnich et al., 2007). Moreover, they evade clearance from immune cells by replicating within phagocytic cells (Glasser et al., 2001), thus promoting their survival below the protective epithelial barrier. These IBD-related E. coli isolates are not amongst the classically known diarrheagenic E. coli, but includes e.g. the prototypic adherent-invasive E. coli (AIEC) strain LF82 (Glasser et al., 2001).

IBD-associated E. coli might promote their establishment in the gut by overgrowing the commensal microbiota via adaption to environmental changes such as inflammation. Thus, instead of being cleared by the host inflammation, it is speculated that these $E$. coli could take advantage of the inflammatory environment and promote chronic gut inflammation. Whether E. coli and/or other microbes take part in the early inflammation or act secondary in the disease progression of $C D$ and UC 
is however unknown. Nonetheless, the bacteria-induced host inflammation significantly affects the host barrier integrity. During homeostasis, the interplay between bacteria and dendritic cells (DCs) is mainly accomplished upon $\mathrm{M}$ cell entry. When the epithelial barrier fails to protect the organism against invading microbes, the immune cells located in the intestinal lamina propria become crucial in preventing systemic spread of the infection. However, infections that evade efficient immune clearance contribute to an on-going active inflammatory response. DCs may be regarded as central for induction of sustained gut inflammation in IBD as they are key controllers in the activation of bacteriaspecific T cells that continuously infiltrate lamina propria during the disease process.

The inflammatory pathophysiological mechanisms of CD and UC seem to differ. $C D$ is characterized by a typical Th1 and Th17 response based on enhanced levels of the cardinal cytokines IFN- $\gamma$ (Th1) and IL17A (Th17), which is activated by lymph node-located DCs that produce IL-12p70 and IFN- $\gamma$ (Th1) and IL-1ß, IL-6 and IL-23 (Th17) (Sakuraba et al., 2009). A prolonged Th1/Th17 activity together with increased levels of pro-inflammatory cytokines (TNF- $\alpha$, IL-1 $\beta$, IL-6) will result in exacerbated epithelial damage and tissue injuries. Collectively, these mechanisms may drive an on-going translocation of enteric bacteria to lamina propria. IBD-associated E. coli could be potent inducers of Th1/Th17propagating DCs due to their display of various pathogen-associated molecular patterns (e.g. lipopolysaccharide (LPS), lipoproteins and peptidoglycan) that activate the expression of immunerelated genes including IL-12p70, IL-23 and IL-6, which drive the Th1/Th17 expansion (Rallabhandi et al., 2008; Roses et al., 2008). Thus, IBD-associated E. coli are plausible drivers of pro-inflammatory response factors related to the Th1/Th17-associated CD pathogenesis. Conversely, the predominant immune phenotype of UC is manifested by a Th2 effector response of high IL-5 and IL-13 levels (Fuss 
et al., 2004; Fuss et al., 1996). IL-13 may play a role in the disease process, as it is recognized to augment e.g. the cytotoxic effect of natural killer T cells on host cells, thereby causing severe tissue damage (Heller et al., 2005). These underlying differences in the inflammatory pathophysiological mechanisms of CD and UC indicate that divergent factors, e.g. different microbes, might be involved in disease manifestation.

Many studies report on changes in the microbial ecosystem in CD and UC patients compared to healthy individuals, but only a few have looked at the functional importance of these observations (Barnich et al., 2007; Darfeuille-Michaud et al., 2004). In the present study, we performed a functional comparison of the host response to prototypic IBD-strains; CD-associated LF82, and UC-associated 691-04A, E. coli Nissle 1917 (ECN) as well as a number of clinical isolates from IBD patients and healthy controls. We used two cell models: human monocyte-derived DCs (moDCs) generated from healthy blood donors and fully differentiated intestinal epithelial cells (Caco-2). Cells were exposed to viable $E$. coli isolates at different bacteria-to-cell ratios to compare the functional attributes of these bacteria. From the cytopathic nature of two isolates from UC patients presented here, we suggest that colonisation with specific non-diarrheagenic $E$. coli promote intestinal barrier leakage and profound intestinal inflammation. Yet, other E. coli as LF82, ECN and the remaining non-diarrheagenic E. coli IBD isolates hold notorious pro-inflammatory characteristics that can progress inflammation in case of intestinal barrier leakage. The molecular attributes within E. coli that determines these differences are yet to be defined. 


\section{Materials and Methods}

\section{Isolation of clinical $E$. coli from human biopsies and faecal samples}

Permission for the study was obtained from the Regional Ethics Committee for Copenhagen County Hospitals (Permission no. H-C-2009-055) and all participants gave their informed written consent. Patients with IBD were diagnosed according to standardized criteria (Langholz et al., 1994; Munkholm et al., 1992). The enrolled healthy control had no history of gastrointestinal disease. Biopsies were taken during videoendoscopy from the ileum and colon with sterile forceps, then directly transferred to Statens Serum Institut (SSI) Transport Medium, and transported to SSI at $4^{\circ} \mathrm{C}$. Immediately upon arrival, biopsies were inoculated on SSI enteric medium (Blom et al., 1999) and incubated at $37^{\circ} \mathrm{C}$ overnight. Faecal cultures were grown by suspending $10 \mu \mathrm{l}$ or an amount equivalent to $10 \mu \mathrm{l}$ faeces into $2 \mathrm{ml}$ of PBS (pH=7.38). The suspension was mixed, and $10 \mu \mathrm{l}$ was plated on SSI enteric medium (Blom et al., 1999) and incubated at $37^{\circ} \mathrm{C}$ overnight. The plates from overnight incubation were examined for colony characteristics, size, and colour of the cultured organisms. Colonies with characteristic features of E. coli and of different morphology (4-5 colonies per sample site when applicable) were chosen for further identification using PGUA test and Minibact E test (Statens Serum Institut, Copenhagen, Denmark) (Kjaeldgaard et al., 1986).

\section{E. coli characterization}

Verified $E$. coli isolates were further characterised genetically by determination of phylogenetic groups ( $\mathrm{A}, \mathrm{B} 1, \mathrm{~B} 2$ and $\mathrm{D})$ based on genes chuA, YjaA and an anonymous DNA fragment, using primers and conditions exactly as described by Clermont et al., 2000. Virulence genes of common E. coli 
diarrheagenic (DEC) pathotypes (VTEC, EPEC, ETEC, EAEC) were detected by DNA probe-hybridization assays: verocytotoxin genes ( $v t x 1, v t \times 2, s a a, e h x A)$, intimin (eae), bundle-forming pili (bfpA), EAST1 (astA), marker for enteroaggregative $E$. coli (aatA, aggR, aaiC), heat labile and heat stabile toxin (/t and st) and marker for diffuse adherence (SLM862) as described previously (Olesen et al., 2005).

Previously, all IBD strains were tested for invasive genes (Vejborg et al., 2011). Sensitivity to Gentamicin of the clinical E. coli strains was tested by disc diffusion assay (Rosco $10 \mathrm{ug}$ ). Invasion ability was tested in a Caco-2 adhesion cell assay.

The prototypic AIEC LF82 isolated from a CD patient was kindly provided by Dr. Arlette DarfeuilleMichaud, Université, d’Auvergne, France. The probiotic bacterium E. coli Nissle 1917 (O6:K5:H1) (ECN) was obtained from Statens Serum Institut, Copenhagen, Denmark. E. coli strains 691-04A and 311-05 have previously been designated p19a and p22, respectively (Petersen et al., 2009, Vejborg et al 2011).

\section{Generation of monocyte-derived dendritic cells}

Monocyte-derived dendritic cells (moDCs) were differentiated from CD14+ monocytic cells obtained from buffy coats of healthy donors (Rigshospitalet, Denmark) as described previously (Sondergaard and Brix, 2012). Briefly, peripheral blood human mononuclear cells were isolated by Ficoll Paque PLUS (GE Healthcare Bio-Sciences AB, Uppsala, Sweden) density gradient centrifugation and CD14+ cells were positively selected by magnetic CD14+ based Microbeads (MACS, Miltenyi Biotec, Bergisch Gladbach, Germany) on a LS Macs separation-column (Miltenyi Biotec). $2 \times 10^{6} /$ well CD14+ monocytes were cultured in 6-well-plates (Nunc, Roskilde, Denmark) for 6 days in RPMI 1640 (Lonza, Vallensbæk, 
Copenhagen) supplemented with $10 \%$ heat-inactivated foetal bovine serum, $1 \%$ penicillinstreptomycin, 1\% L-glutamine (all Lonza, Vallensbæk, Copenhagen) and differentiated to moDCs by adding $30 \mathrm{ng} / \mathrm{mL}$ IL-4 (CellGenix, Freiburg, Germany) and $20 \mathrm{ng} / \mathrm{mL}$ GM-CSF (CellGenix) in the culture medium. On day 3 , half of the cell culture medium was replaced with fresh medium containing 60 $\mathrm{ng} / \mathrm{mL}$ IL-4 and $40 \mathrm{ng} / \mathrm{mL}$ GM-CSF. On day 6, moDCs were tested for expression of CD1a and CD14 by flow cytometry to ascertain for differentiation of monocytes into moDCs. moDC cultures used for the study were >95\% CD1a-positive.

\section{Culture of intestinal epithelial cell line}

Caco-2 cells (ATCC-HTB-37, Manassas, USA) (passage numbers $p=22-26$ ) were cultured in Dulbecco's modified Eagle medium (DMEM, Lonza) supplemented with $10 \%$ heat-inactivated foetal bovine serum, $1 \%$ penicillin-streptomycin, $1 \%$ non-essential amino acids, and $1 \%$ L-glutamine (all Lonza). Cells were seeded in 12-well tissue-culture treated polycarbonate transwell inserts (12 mm diameter, 0.4 $\mu \mathrm{m}$ pore size, Costar, Corning, NY) at a cell density of $4.25 \times 10^{4}$ cells/well. Medium was replaced every 2 nd to 3 rd day. On day 21, cells were fully differentiated and were used for co-culture with bacteria.

\section{Host cell stimulation with viable $E$. coli}

E. coli were grown overnight in Luria-Bertani medium to stationary phase. Bacteria were then washed twice in PBS ( $\mathrm{pH}=7.2)$ prior to addition to host cells. Multiplicity of infection (MOI) was adjusted using a pre-determined optical density-to-colony-correlation factor for each isolate. 
A total of $6 \times 10^{5}$ moDCs per well were seeded in 48-well suspension plates (Greiner Bio-One, Monroe, North Carolina, USA) in antibiotic-free RPMI 1640 supplemented with $10 \%$ heat-inactivated foetal bovine serum, and $1 \%$ L-glutamine (all Lonza). Cells were co-cultured with live E. coli at different MOIs: 1,5 and 10, tested in a blinded fashion. LPS $(1 \mu \mathrm{g} / \mathrm{mL}$ ) from E. coli O26:B6 (SigmaAldrich) was used as a positive control. Following $1 \mathrm{~h}$ of stimulation, gentamicin $(50 \mu \mathrm{g} / \mathrm{mL}$, Sigma Aldrich, Steinham, Germany) was added to kill remaining extracellular bacteria. moDCs were incubated with bacteria for a further $19 \mathrm{~h}$. Supernatants were harvested after $20 \mathrm{~h}$, and the cell viability and intracellular production of reactive oxygen species (ROS) were both analysed by flow cytometry.

The monolayer of differentiated Caco- 2 cells in transwells was washed in $37^{\circ} \mathrm{C}$ pre-warmed PBS and replaced with fresh pre-warmed culture medium without antibiotics. The number of differentiated Caco- 2 cells was determined in one to three wells by cell counting (NucleoCounter, Chemometec, Allerød, Denmark). On average, $6 \times 10^{5}$ viable cells were present in the transwells, and stimulated with live $E$. coli at different MOls: 100, 500 and 1000. After $1 \mathrm{~h}$ of stimulation, $50 \mu \mathrm{g} / \mathrm{mL}$ (MOI 100) or $100 \mu \mathrm{g} / \mathrm{mL}$ gentamicin (MOI 500, 1000) was added to kill remaining extracellular bacteria. The cocultivation continued for a further $17 \mathrm{~h}$ before measurement of cell viability and intracellular production of ROS. Cell supernatants were stored at $-80^{\circ} \mathrm{C}$ until chemokine- and cytokine analysis by ELISA. 
Before inclusion of gentamicin, we examined the dose-dependent effects on cytokine production and cell viability in LPS-stimulated and medium-treated cells, and found no effects at the given gentamicin concentrations. Accordingly, gentamicin was added to all treatments, including medium-treated cells.

\section{Flow cytometry}

moDCs were stained with 2'-7'-Dichlorodihydrofluorescein diacetate (DCFH-DA) (Invitrogen, Eugene, OR, USA) for measurement of intracellular ROS. Briefly, DCFH-DA is a non-fluorescent stain that is taken up by cells and oxidized to fluorescent dichlorofluorescein (DCF) by intracellular esterases in the presence of ROS. SYTOX ${ }^{\circledR}$ AADvanced ${ }^{\mathrm{TM}}$ (AAD) (Invitrogen) was used to assess cell viability, as it binds to the nucleic acids of dead cells. Cells were stained with $5 \mu \mathrm{M}$ DCFH-DA for 45 min and with $0.2 \mu \mathrm{L}$ SYTOX-AAD/120,000 cells just prior to analysis. Flow cytometry analysis was carried out on a BD FACSCanto II Flow Cytometer (Franklin Lakes, NJ, USA) and data were further analysed with FlowJo software (Tree Star, Inc. / FlowJo LLC, Ashland, OR, USA).

\section{Cytokine analysis of moDC and Caco-2 supernatants}

Cytokines and chemokines in the cell supernatants were determined by ELISA according to the manufacturer's instructions. The following kits were used: IL-8 (BD Biosciences), TNF- $\alpha$, IL-6, IL-1 $\beta$, IL12p70, IL-10 (all R\&D Systems, Minneapolis, MN, USA), IL-18 and IL-23 (both eBioscience).

\section{Statistics}

Data are based on moDCs generated from 11 healthy donors, except for data on C311-05 that was tested on the five last donors. Data on cellular viability and ROS production are based on five 
individual donors. All displayed bacteria treatments were tested on cells from each donor. Nonparametric statistics was used to test statistical differences between treatments by use of GraphPad Prism 5 (GraphPad Software, San Diego, CA, USA). 


\section{Results}

\section{Characterization of intestinal E. coli isolates}

The site of isolation, patient group and phylogeny characteristics for LF82, 691-04A, ECN and the thirteen clinical E. coli isolates are presented in Table 1. It is worth pointing out that the patients were colonised by only one or two $E$ coli types, indicating the possible importance of the colonising strains in the development of disease.

All patients diagnosed with UC were colonised with E. coli from the phylogenetic group B2. Isolates from $C D$ patients were of the mixed phylogenetic groups $A, B 2$ and D. Groups B2 and D were previously associated with IBD (Kotlowski et al., 2007). Based on molecular assays, no verotoxinproducing genes, invasion genes or other common diarrheagenic virulence genes were detected amongst the IBD E. coli isolates (Table 1), and therefore we characterise the isolates as nondiarrheagenic E. coli.

\section{Response profiles in dendritic cells challenged with non-diarrheagenic E. coli}

MoDCs were cultured with viable $E$. coli at three bacteria-to-cell ratios (1:1 (MOI 1), 5:1 (MOI 5) and 10:1 (MOI 10)), followed by measurement of secreted cytokines and cell viability upon $20 \mathrm{~h}$ of stimulation. LPS and unstimulated (medium) moDCs were included as reference controls. All bacteria were found to induce a dose-dependent production of four out of the seven selected cytokines: IL-1 $\beta$, IL-23, IL-12p70 and IL-10; displayed here for LF82 (Fig. 1A). Increasing the bacterial load from MOI 1 to 10 correlated with a rise in nearly all pro-inflammatory cytokines, except for IL- 6 and TNF- $\alpha$, which were generally highly expressed also at MOI 1, and for the inflammasome-derived cytokine IL-18 
which was only slightly induced in LF82-exposed moDCs (Fig. 1A). As expected, IL-1 $\beta$ and IL-18 were not induced by pure LPS in moDCs, since cytokine levels for LPS correspond to that of unstimulated cells (representing cytokine detection limits).

We made spider-web plots to compare the cytokine secretion patterns for all bacteria-to-cell ratios of the viable $E$. coli isolates, and found that cytokine profiles for LF82 corresponded to that of ECN (Fig. 1B), and also to ten of the new E. coli intestinal isolates (supplementary Fig. 1A). Notably, we identified a disparate cytokine pattern for C691-04A (Fig. 1B), single-handedly resembling that of the intestinal isolate C311-05 (supplementary Fig. 1B); and only C311-05 was found to enhance IL-18 levels similar to C691-04A (Fig. 1C).

When focusing on the overall abundances of the seven cytokines from E. coli-exposed moDCs, we found great differences between C691-04A and LF82 (Fig. 1D). For C691-04A at MOI 10, IL-6 and TNF$\alpha$ each made up $30-40 \%$ of the measured cytokines, IL-18 15\%, and IL-23 8\%, while IL-12p70 and IL-10 were about $3 \%$ of total cytokines, and IL-1 $\beta$ was almost absent. For LF82 at MOI 10, the moDC cytokine pattern was tipped towards approx. 50\% TNF- $\alpha$, 30\% IL-6, 9\% IL-10, 5\% IL-23, 5\% IL-12p70 and little of the caspase-1-induced IL-1ß (1\%) and IL-18 (0.06\%) (Fig. 1D), which remained almost constant at all three MOls. For C691-04A it appeared that IL-18 levels increased with increasing MOI, while IL-12p70 and IL-10 levels diminished (Fig. 1D). When addressing the actual concentrations of secreted cytokines from LF82- and C691-04A-cultured moDCs, it was evident that the amount of all secreted cytokines was gradually reduced with increased MOI for C691-04A (Fig. 1E), which, together with increased IL-18 levels, indicates that cytopathic effects may be in play. 
Viability of dendritic cells challenged with non-diarrheagenic E. coli

The viability of moDCs at $20 \mathrm{~h}$ after LF82 exposure changed in a MOI-dependent manner with a slight decrease in viability from MOI 1 (approx. 55\% viable cells) to MOI 10 (approx. $40 \%$ viable cells), but the percentage of viable cells was still comparable with that of unstimulated and LPS-treated cells, even at increasing MOIs (Fig. 2A). Viability was comparable for all bacteria except for C691-04A where it dropped to less than $10 \%$ at MOI 5 and 10 (Fig. 2B).

Altogether, moDCs responded similarly to CD-associated LF82 and commensal ECN, as well as ten of the new E. coli isolates that covered both B2 and other phylogenetic groups (Table 1). As C691-04A promoted enhanced IL-18 and reduced viability at $20 \mathrm{~h}$ post challenge, we further examined changes in the cellular viability at $4 \mathrm{~h}$ post exposure to study initial differences in the moDC response against IBDassociated E. coli strains.

\section{Early changes in cellular viability dynamics of C691-04A-exposed dendritic cells}

The cellular regulations that might predispose to the noticeably higher moDC mortality mediated by C691-04A at $20 \mathrm{~h}$ post challenge was studied by examining viability versus cell death modes in moDCs at $4 \mathrm{~h}$ post challenge. The dead-marker stain AAD was applied to distinguish between dead and viable cells by flow cytometry, and moreover to classify cell subpopulations that do not strictly group into these two categories. By use of AAD versus side scatter (SSC) biplots, we identified five distinguishable moDC subpopulations that were designated: $V, D 1, D 2, D 3$ and $D 4$ cells, as displayed for LF82-exposed 
moDCs (Fig. 3A). Cells termed, $V$, were viable (negative for AAD staining, Fig. 3A), and exhibited higher forward-scatter (FSC, i.e. cell size) properties compared to the other cells (Fig. 3B). Cells in D1 were negative for AAD and in combination with a low FSC versus SSC, we identified these cells to be apoptotic (also confirmed by stimulation of moDCs with the apoptosis-inducing drug camptothecin that induced $>50 \%$ D1 cells, and by Vybrant DyeCycle Violet/SYTOX AADvanced staining to identify FSC-SSC properties of apoptotic cells (data not shown)). D2- and D3 cells displayed a low but indistinguishable FSC, suggesting that these cells were dead. Cells of $D 2$ stained negative for AAD, and in combination with the low FSC, D2 makes up dead cells probably without DNA due to degradation. $D 3$ cells were positive for $A A D$, i.e. dead cells. $D 4$ cells were also identified as dead, as they stained positive for AAD, but due to their SSC resembling that of viable cells, $D 4$ cells seem to be less dysfunctional than $D 3$ and $D 2$. By this procedure, we defined cells in $D 2, D 3$ and $D 4$ to represent necrotic cells that are disparate from the apoptotic cells in D1. ECN (Fig. 3C, D) and the 10 newly isolated E. coli isolates listed in Table 1 promoted the same cellular death modes and percentages of these as presented for LF82 (data not shown). However, the subpopulations induced by C691-04A at MOI 1, 5 and 10, and by C311-05 at MOI 5 and 10 changed considerably as compared to those identified in LF82- and ECN-exposed cells (Fig. 3), making it almost impossible to identify the same subpopulations as for LF82 and ECN. The differences are therefore best described by simply looking at the dynamic changes in the biplots showing the distribution of moDC subpopulations induced by C691-04A at different MOIs; as compared to the other E. coli at similar MOIs. Specifically, we found overall changes in the properties of the $V$ population (lower SSC), as well as increased apoptosis (D1) already at MOI 1 with C691-04A, and at MOI 5 and 10 with C311-05 (Fig. 3D). The apparent changes in 
FSC-SSC-properties of the $V$ population in C691-04A-exposed moDCs (Fig. 3D) indicated that the $V$ subpopulation from C691-04A-exposed moDCs might include cells that hold other characteristics than solely viable cells.

The cytopathic properties of $E$. coli C691-04A promote induction of a distinct dendritic cell subtype

To examine the characteristics of the $V$ subpopulation, we first compared the early changes of the live/dead cell dynamics at $4 \mathrm{~h}$ induced by C691-04A and LF82 at MOI 1 (Fig. 4A). The $V$ subpopulation that was apparent $4 \mathrm{~h}$ post challenge could be divided into two subpopulations, designated $D 5$ and V0, based on their FSC-properties. D5 cells of C691-04A displayed a lower FSC than V0, they were moreover negative for AAD-staining and showed a dispersed SSC with the major part of cells having a low SSC, resembling that of dead cells (Fig. 4A). Noticeably, the $D 5$ population was only induced by C691-04A, and marginally by C311-05 at MOI 5 and 10 (the SSC-high population in between $V$ and D4 in Fig. 3D), but not by LF82 (Fig. 4A).

When comparing the cellular characteristics induced at 4 and $20 \mathrm{~h}$ post challenge with C691-04A at MOI 1, 5 and 10, it was apparent that the $D 5$ subpopulation was visible only upon $4 \mathrm{~h}$ challenge (Fig. 4B). After $20 \mathrm{~h}$, the majority of moDCs were identified as dead based on low viability ( $<10 \%$ at MOI 5 and 10) and an increase in AAD-stained cells, mainly D3 cells ( $20 \%$ and $55 \%$ at MOI 10 after 4 and $20 \mathrm{~h}$ of stimulation, respectively, data not shown for $4 \mathrm{~h}$ ). It was also evident that the SSC-properties of $D 5$ cells were MOI-dependent at $4 \mathrm{~h}$ post challenge, as low SSC was seen at MOI 1 and 5, while the few viable cells (V) at MOI 10, displayed higher SSC properties (Fig. 4B). Since differences in attributes of 
the $4 \mathrm{~h}$ stimulated moDC subpopulations seem to relate to the later cellular fate, i.e. cytopathicity or survival/containment of bacteria, we also looked into the cellular ability to produce reactive oxygen species (ROS), as ROS are recognized to take part in bacterial breakdown in phagocytic cells. High ROS production can therefore be regarded as a measure of metabolically active cells that are able to handle bacterial exposure.

\section{Production of reactive oxygen species in dendritic cells challenged with non-diarrheagenic E. coli}

Intracellular ROS production in each identified subpopulation from Fig. 4B is illustrated at 4 and $20 \mathrm{~h}$ post challenge with C691-04A (Fig. 5A). Cells that were AAD-negative ( $V, D 5$ and $D 1$ ) were also ROSproducing, thus representing metabolically active and intact cells. However, the highest levels of ROS were produced from $V(4$ and $20 \mathrm{~h})$ and $D 5(4 \mathrm{~h})$ cells (Fig. 5A). On the contrary, D2, D3 and D4 cells were low in ROS, likely as a consequence of their lack of cellular intactness (i.e. membrane leakiness is a prerequisite for positive AAD-staining). Intracellular ROS was measurable both at 4 and $20 \mathrm{~h}$ post challenge, implying that ROS production is not only an early-induced host effect. Moreover, when comparing intracellular ROS induced by LF82, ECN, C691-04A and C311-05 in viable cells (V), there were no apparent differences at any tested MOI (Fig. 5B). From these observations, it seems that ROS production might be a general host defence mechanism provoked independently of the pathogenicity of the single $E$. coli strain, and thus not a specific phenomenon linked to bacterial cytopathic effects.

Based on the current analysis of changes in moDC subpopulations, and not specifically their ROS characteristics, it is noticeable that early appearance of the D5 subpopulation promoted by C691-04A 
at all MOIs, and by C311-05 at MOI 5 and 10 (supplementary Fig. 2), relate to the attribute of these bacteria to induce high IL-18 levels and increased overall cell death at $20 \mathrm{~h}$ post challenge (summarized in Table 2).

\section{E. coli C691-04A and ECN have equal cytopathic properties in intestinal epithelial cells at high MOI}

The similarity in the pro-inflammatory cytokine response induced in moDCs upon challenge with most clinical $E$. coli strains implies that all the $E$. coli are able to induce a profound immune activation in moDCs if they get into contact with them i.e. by breaching the epithelium or crossing via M cells. Breaching via the epithelium might be induced by bacteria that mediate a cytopathic effect on the epithelium; manifested in vitro by impaired viability, coupled with excretion of IL-18. As C691-04A showed these specific features in moDCs, we speculated if such cytopathic effects were also evident upon its interaction with epithelial cells.

Intestinal epithelial cells (IECS) were grown to confluence in transwells and then exposed to C691-04A at $\mathrm{MOI}$ 100, 500 and 1000, followed by comparison of the apical cytokine response to the response induced by LF82 and ECN. Five different cytokines were evaluated: IL-18, IL-8, IL-1 $\beta$, TSLP and TNF- $\alpha$, but only IL-18 and IL-8 were detectable.

IL-18 was secreted at high bacteria-to-cell ratios (MOI 1000) by all three bacteria. Noticeable, C69104A and ECN mediated up to 3-fold higher IL-18 secretion compared to LF82 at MOI 1000, suggesting a different potency amongst the bacteria at high MOI (Fig. 6A). The IL-8 decreased as MOI increased. 
At MOI 1000, C691-04A and ECN did not induce IL-8 secretion, in contrast to LF82 (Fig. 6B). The cell mortality followed the cytokine pattern, as there was a clear drop in viability above MOI 500, which was most profoundly observed upon challenge with C691-04A and ECN (Fig. 6C). IECs were still metabolically active in regard to intracellular ROS production up to $\mathrm{MOI} 1000$ for LF82, and up to $\mathrm{MOI}$ 500 for C691-04A and ECN (Fig. 6D), where viability also declined dramatically for the two latter. These data demonstrate that at very high cell-to-bacteria ratios C691-04A and ECN are both capable of promoting inflammation-induced cell death in IECs, and that LF82 is less potent in provoking cytopathic effects in IECs at high MOI. 


\section{Discussion}

The functional comparative analysis of non-diarrheagenic E. coli clinical isolates showed that $E$. coli in general, regardless of intestinal location, were able to promote a characteristic, foreseeable, proinflammatory cytokine response in moDCs. Besides, two $E$. coli isolates also revealed distinct cytopathic features mainly in moDCs but also in IEC at high bacterial numbers.

The most common fingerprint in E. coli-challenged moDCs was high secretion of TNF- $\alpha$, IL-6, IL-10, but also noticeable levels of the Th1-polarizing cytokine IL-12p70 and Th17-expanding IL-23. These cytokines make up a predictable outcome in E. coli-exposed moDCs based on the presence of the immunostimulatory ligands LPS, peptidoglycan and lipoproteins in E. coli. However, it is noticeable that the two UC-derived E. coli strains C691-04A and C311-05 evoked up to 18-times less total cytokines at MOI 10, while increasing the levels of IL-18 more than 15 -fold compared to the remaining E. coli strains. Moreover the two UC-derived strains reduced overall cell survival, and changed the cellular death modes in a manner essentially differing from the other E. coli isolates. Particularly, E. coli C691-04A was observed to mediate specific inflammation-induced killing at high bacteria-to-cell ratios in both moDCs and IECs. Such properties may impair barrier function, promote bacterial translocation into the gut lamina propria, and thereby cause activation of phagocytes, including DCs, as shown here. Since moDCs were profoundly affected by C691-04A at low bacteria-to-cell ratios, the explicit behaviour of this $E$. coli strain could be speculated to mediate specific pathophysiological processes relating to IBD, or more specifically to the disease process within the UC patient from whom it was isolated. The distinct properties of C691-04A on moDCs were likewise observed by E. coli C311-05, although less profound effects were seen at MOI 10. 
The observed host-phenotype induced by UC-derived C691-04A and C311-05 has to our knowledge not previously been described as a feature of IBD-associated E. coli. Today, the features of AIEC, i.e. 1) adhesion to IECS, 2) invasion in IECs and 3) escape from host clearance (Boudeau et al., 1999; Darfeuille-Michaud et al., 2004; Glasser et al., 2001), are considered as cardinal characteristics, used to identify functional effects of E. coli in relation to IBD, and in particular CD. Here, we demonstrate that parameters relating to innate immune activation and cell death, specifically the inflammatory profiles in moDCs, as well as the type of cell death may be important for judging $E$. coli pathogenicity in the host. Thus, new properties within IBD-associated $E$. coli have been described herein, and we suggest that these attributes are likely to contribute in the processes resulting in epithelium breakdown, and the ongoing elicitation of inflammatory leukocytes and cytokines characteristic for both UC and CD.

IL-18 is an important cytokine bridging the innate and adaptive immune system by differentiating and activating IL-12p70-promoted Th1 cells (Dinarello, 1999). The significance of IL-18 in the inflammatory progression of IBD has been reported by several studies (Leach et al., 2008; Pizarro et al., 1999), and the effect of neutralizing IL-18 in animal models, has shown to reduce disease-driven inflammation (Sivakumar et al., 2002). Secretion of mature IL-18 is based on inflammasome activation involving a caspase-1-dependent mechanism initiated as cells undergo inflammation-induced cell death in response to host-depriving signals. This fate of cell death is known as pyroptosis, and is believed to become effective when the host cannot clear the bacteria through the common non-inflammatory death route i.e. apoptosis (Ashida et al., 2011). As already reported for Salmonella enterica serovar Typhimurium and Staphylococcus aureus (Miao et al., 2010; Soong et al., 2012), it is likely that E. coli 
C691-04A and C311-05 also induce host cell death via the pyroptotic way, which thereby would explain the high IL-18 levels, the increase in cell mortality and the early distinct cell death modes induced in moDCs exposed to these $E$. coli strains. Based on these observations we speculate that the distinct moDC population, D5, mediated by C691-04A and C311-05, but not the other $E$. coli isolates, is an initial identifier for cells undergoing pyroptosis. To conclude on this, more studies are needed that focus on the mechanisms determining the distinct host cell death, but also on the specific attributes of E. coli C691-04A and C311-05 that makes them cytotoxic.

We also observed an early increase in the apoptotic cell population (D1) at stimulation with C691-04A and C311-05, implying that other death processes seem to proceed in the early phase of interaction with moDCs. It has previously been shown that Yersinia spp. elicit apoptosis in the early phase of an infection (Ashida et al., 2011), likely as a strategy to prevent inflammation-induced host clearance, and thereby gain time to establish prolonged infection (Ashida et al., 2011).

Besides mediating a similar and distinct host response, the two E. coli isolates C691-04A and C311-05, also have in common that they are isolated from UC patients. Although few in numbers, this observation brings forward that some $E$. coli from UC patients are able to induce a distinct cytopathicity dissimilar from other $E$. coli isolates. However, it is evident that many more isolates will need characterization to settle whether specific $E$. coli strains might be selectively involved in initiation of UC.

It has for long been known that Th1/Th17-expansion is a hallmark for CD lesions (Sakuraba et al., 2009), whereas Th2 is more dominant in UC (Fuss et al., 1996). Based on the current observations, it is 
striking that the cytokine profile promoted by the two unique UC-associated E. coli strains relate to common UC-disease characteristics. While IL-18 in combination with IL-12p70 is known to promote Th1 expansion, the effector function of IL-18 in the absence of IL-12p70 is reported to mediate Th2 progression of naïve T cells (Nakanishi et al., 2001) as well as IL-4 and IL-13 production by basophils, which favours Th2 polarization (Kroeger et al., 2009). That C691-04A in particular, but also C311-05, mediated a cytokine response with high IL-18 and low IL-12p70 at MOI 10 suggests that these two bacteria might hold the potential to promote expansion of Th2 cells rather than Th1, the latter requiring IL-12p70. On the contrary, CD-associated LF82 and the clinical E. coli isolates from CD patients provoked a strong pro-inflammatory response represented by profound TNF- $\alpha$, IL-6, IL-10, high IL-12p70, IL-23 and also IL-1 3 , thus suggesting expansion of Th1 (via IL-12p70) and Th17 (via IL-6, IL-1 $\beta$, IL-23). Hence, although the pathogenesis of CD and UC both involves $E$. coli, it could be speculated that intrinsic differences amongst colonizing $E$. coli in terms of the way of interacting with mODC and IEC might account for the divergence in immunophenotypes identified as characteristics for each disease.

In the literature ECN has been described as a non-pathogenic probiotic $E$. coli with the ability to competitively exclude LF82 adhesion to IECs and to exert anti-inflammatory properties to host cells (Boudeau et al., 2003; Huebner et al., 2011). On the other hand, ECN as well as E. coli LF82 and C69104A all belong to the phylogenetic group B2, which is identified to be a common characteristic of $E$. coli strains isolated from a diseased gut (Petersen et al., 2009). Moreover, ECN is also reported to contain virulence genes commonly found in pathogenic E. coli strains (Grozdanov et al., 2004; Picard et al., 1999) suggesting that it might carry pathogenic potential. The data presented herein support 
the notion that $\mathrm{ECN}$ displays similar immunostimulatory capacity as most other non-diarrheagenic $E$. coli, including CD-derived LF82. Additionally, we have previously shown that ECN is unable to exclude adhesion of LF82 to human ileal and colonic biopsies from CD patients (Jensen et al., 2011). ECN has been used in clinical trials with IBD patients where it is shown to prevent relapse in UC to a similar extent as the anti-inflammatory drug mesalazine in inactive UC (Kruis et al., 2004), whereas no effects of ECN administration has been reported in CD patients (Jonkers et al., 2012). Our previous and present data support that ECN might be ineffective in $C D$, not only due to its inability to outcompete strains such as LF82, but also because it induces inflammation similar to CD-associated strains. However, it still remains to be identified if ECN have a protective role in UC patients for instance by outcompeting strains such as C691-04A or by shifting the cytokine profile induced by strains like C69104A. Still, it should be stressed that our in vitro data revealed a cytopathic effect of ECN in IECs similar to that of C691-04A at MOI 1000, implying that ECN might be able to induce considerable epithelial damage if it is able to expand vigorously in an IBD-milieu.

There are several data showing a disease-relevance of $E$. coli in IBD based on $E$. coli strains being more numerous in IBD compared to healthy guts (Conte et al., 2006; Kotlowski et al., 2007; MartinezMedina et al., 2006). Our present functional study add further information to these prior findings by demonstrating that some of the clinical E. coli isolates diverge from each other in terms of the phenotype they impose on innate cells of the host. Moreover, we find a notable match between the in vitro based cytokine response profile from moDC towards two of the UC-isolated strains versus the remaining E. coli spp. and the prevailing host response Th-phenotype (Th2 vs Th1/Th17) describing UC and $C D$, respectively. At present we have not identified the bacterial virulence factors within $\mathrm{C691-}$ 
04A and C311-05 that account for this difference, but studies are ongoing to clarify their mechanism of action.

Conclusively, this study identifies two functionally different $E$. coli subgroups in IBD-mucosa that induce distinct pro-inflammatory phenotypes in moDCs and in intestinal epithelium at high bacterial numbers. The distinct functional differences within $E$. coli isolates may be an underlying causative mediator for the different pathophysiological representations in UC versus CD lesions. 


\section{Acknowledgements}

We are grateful to the participants consenting to this study and to Dr. Jakob Hendel, MD, PhD for performing the ileocolonoscopy. We also thank Dr. Arlette Darfeuille-Michaud for providing the AIEC-

LF82 strain, and Susanne Jespersen, Statens Serum Institut, Denmark for typing the E. coli strains. 


\section{References}

Ashida, H., Mimuro, H., Ogawa, M., Kobayashi, T., Sanada, T., Kim, M., Sasakawa, C., 2011. Hostpathogen interactions Cell death and infection: A double-edged sword for host and pathogen survival. J Cell Biol 195, 931-942.

Barnich, N., Carvalho, F.A., Glasser, A.L., Darcha, C., Jantscheff, P., Allez, M., Peeters, H., Bommelaer, G., Desreumaux, P., Colombel, J.F., Darfeuille-Michaud, A., 2007. CEACAM6 acts as a receptor for adherent-invasive E. coli, supporting ileal mucosa colonization in Crohn disease. J Clin Invest 117, 1566-1574.

Blom, M., Meyer, A., Gerner-Smidt, P., Gaarslev, K., Espersen, F., 1999. Evaluation of Statens Serum Institut enteric medium for detection of enteric pathogens. J Clin Microbiol 37, 2312-2316.

Boudeau, J., Glasser, A.L., Julien, S., Colombel, J.F., Darfeuille-Michaud, A., 2003. Inhibitory effect of probiotic Escherichia coli strain Nissle 1917 on adhesion to and invasion of intestinal epithelial cells by adherent-invasive E-coli strains isolated from patients with Crohn's disease. Aliment Pharmacol Ther $18,45-56$.

Boudeau, J., Glasser, A.L., Masseret, E., Joly, B., Darfeuille-Michaud, A., 1999. Invasive ability of an Escherichia coli strain isolated from the ileal mucosa of a patient with Crohn's disease. Infect Immun $67,4499-4509$.

Clermont, O., Bonacorsi, S., Bingen, E., 2000. Rapid and simple determination of the Escherichia coli phylogenetic group. Appl Environ Microbiol 66, 4555-4558.

Conte, M.P., Schippa, S., Zamboni, I., Penta, M., Chiarini, F., Seganti, L., Osborn, J., Falconieri, P., Borrelli, O., Cucchiara, S., 2006. Gut-associated bacterial microbiota in paediatric patients with inflammatory bowel disease. Gut 55, 1760-1767.

Darfeuille-Michaud, A., Boudeau, J., Bulois, P., Neut, C., Glasser, A.L., Barnich, N., Bringer, M.A., Swidsinski, A., Beaugerie, L., Colombel, J.F., 2004. High prevalence of adherent-invasive Escherichia coli associated with ileal mucosa in Crohn's disease. Gastroenterology 127, 412-421.

Dinarello, C.A., 1999. IL-18: A T-H1-inducing, proinflammatory cytokine and new member of the IL-1 family. J Allergy Clin Immunol 103, 11-24.

Fuss, I.J., Heller, F., Boirivant, M., Leon, F., Yoshida, M., Fichtner-Feigl, S., Yang, Z.Q., Exley, M., Kitani, A., Blumberg, R.S., Mannon, P., Strober, W., 2004. Nonclassical CD1d-restricted NK T cells that produce IL-13 characterize an atypical Th2 response in ulcerative colitis. J Clin Invest 113, 1490-1497.

Fuss, I.J., Neurath, M., Boirivant, M., Klein, J.S., delaMotte, C., Strong, S.A., Fiocchi, C., Strober, W., 1996. Disparate CD4(+) lamina propria (LP) lymphokine secretion profiles in inflammatory bowel 
disease - Crohn's disease LP cells manifest increased secretion of IFN-gamma, whereas ulcerative colitis LP cells manifest increased secretion of IL-5. J Immunol 157, 1261-1270.

Glasser, A.L., Boudeau, G., Barnich, N., Perruchot, M.H., Colombel, J.F., Darfeuille-Michaud, A., 2001. Adherent invasive Escherichia coli strains from patients with Crohn's disease survive and replicate within macrophages without inducing host cell death. Infect Immun 69, 5529-5537.

Grozdanov, L., Raasch, C., Schulze, J., Sonnenborn, U., Gottschalk, G., Hacker, J., Dobrindt, U., 2004. Analysis of the genome structure of the nonpathogenic probiotic Escherichia coli strain Nissle 1917. J Bacteriol 186, 5432-5441.

Heller, F., Florian, P., Bojarski, C., Richter, J., Christ, M., Hillenbrand, B., Mankertz, J., Gitter, A.H., Burgel, N., Fromm, M., Zeitz, M., Fuss, I., Strober, W., Schulzke, J.D., 2005. Interleukin-13 is the key effector Th2 cytokine in ulcerative colitis that affects epithelial tight junctions, apoptosis, and cell restitution. Gastroenterology 129, 550-564.

Huebner, C., Ding, Y.Y., Petermann, I., Knapp, C., Ferguson, L.R., 2011. The Probiotic Escherichia coli Nissle 1917 Reduces Pathogen Invasion and Modulates Cytokine Expression in Caco-2 Cells Infected with Crohn's Disease-Associated E. coli LF82. Appl Environ Microbiol 77, 2541-2544.

Jensen, S.R., Fink, L.N., Nielsen, O.H., Brynskov, J., Brix, S., 2011. Ex vivo intestinal adhesion of Escherichia coli LF82 in Crohn's disease. Microb Pathog 51, 426-431.

Jonkers, D., Penders, J., Masclee, A., Pierik, M., 2012. Probiotics in the Management of Inflammatory Bowel Disease A Systematic Review of Intervention Studies in Adult Patients. Drugs 72, 803-823.

Kjaeldgaard, P., Nissen, B., Lange, N., Laursen, H., 1986. Evaluation of Minibact, a New System for Rapid Identification of Enterobacteriaceae - Comparison of Minibact, Micro-Id and Api-20e with a Conventional Method as Reference. Acta Path Micro Im B 94, 57-61.

Kotlowski, R., Bernstein, C.N., Sepehri, S., Krause, D.O., 2007. High prevalence of Escherichia coli belonging to the B2+D phylogenetic group in inflammatory bowel disease. Gut 56, 669-675.

Kroeger, K.M., Sullivan, B.M., Locksley, R.M., 2009. IL-18 and IL-33 elicit Th2 cytokines from basophils via a MyD88-and p38 alpha-dependent pathway. J Leukoc Biol 86, 769-778.

Kruis, W., Fric, P., Pokrotnieks, J., Lukas, M., Fixa, B., Kascak, M., Kamm, M.A., Weismueller, J., Beglinger, C., Stolte, M., Wolff, C., Schulze, J., 2004. Maintaining remission of ulcerative colitis with the probiotic Escherichia coli Nissle 1917 is as effective as with standard mesalazine. Gut 53, 16171623.

Langholz, E., Munkholm, P., Davidsen, M., Binder, V., 1994. Course of Ulcerative-Colitis - Analysis of Changes in Disease-Activity over Years. Gastroenterology 107, 3-11. 
Leach, S.T., Messina, I., Lemberg, D.A., Novick, D., Rubenstein, M., Day, A.S., 2008. Local and systemic interleukin-18 and interleukin-18-binding protein in children with inflammatory bowel disease. Inflamm Bowel Dis 14, 68-74.

Lees, C.W., Barrett, J.C., Parkes, M., Satsangi, J., 2011. New IBD genetics: common pathways with other diseases. Gut 60, 1739-1753.

Martinez-Medina, M., Aldeguer, X., Gonzalez-Huix, F., Acero, D., Garcia-Gil, L.J., 2006. Abnormal microbiota composition in the ileocolonic mucosa of Crohn's disease patients as revealed by polymerase chain reaction-denaturing gradient gel electrophoresis. Inflamm Bowel Dis 12, 1136-1145.

Miao, E.A., Leaf, I.A., Treuting, P.M., Mao, D.P., Dors, M., Sarkar, A., Warren, S.E., Wewers, M.D., Aderem, A., 2010. Caspase-1-induced pyroptosis is an innate immune effector mechanism against intracellular bacteria. Nat Immunol 11, 1136-U1194.

Mondot, S., Kang, S., Furet, J.P., de Carcer, D.A., McSweeney, C., Morrison, M., Marteau, P., Dore, J., Leclerc, M., 2011. Highlighting New Phylogenetic Specificities of Crohn's Disease Microbiota. Inflamm Bowel Dis 17, 185-192.

Munkholm, P., Langholz, E., Nielsen, O.H., Kreiner, S., Binder, V., 1992. Incidence and Prevalence of Crohns-Disease in the County of Copenhagen, 1962-87 - a Sixfold Increase in Incidence. Scand J Gastroenterol 27, 609-614.

Nakanishi, K., Yoshimoto, T., Tsutsui, H., Okamura, H., 2001. Interleukin-18 is a unique cytokine that stimulates both Th1 and Th2 responses depending on its cytokine milieu. Cytokine Growth Factor Rev $12,53-72$.

Olesen, B., Neimann, J., Bottiger, B., Ethelberg, S., Schiellerup, P., Jensen, C., Helms, M., Scheutz, F., Olsen, K.E.P., Krogfelt, K., Petersen, E., Molbak, K., Gerner-Smidt, P., 2005. Etiology of diarrhea in young children in Denmark: a case-control study. J Clin Microbiol 43, 3636-3641.

Petersen, A.M., Nielsen, E.M., Litrup, E., Brynskov, J., Mirsepasi, H., Krogfelt, K.A., 2009. A phylogenetic group of Escherichia coli associated with active left-sided Inflammatory Bowel Disease. BMC microbiol 9, 171.

Picard, B., Garcia, J.S., Gouriou, S., Duriez, P., Brahimi, N., Bingen, E., Elion, J., Denamur, E., 1999. The link between phylogeny and virulence in Escherichia coli extraintestinal infection. Infect Immun 67, 546-553.

Pizarro, T.T., Michie, M.H., Bentz, M., Woraratanadharm, J., Smith, M.F., Foley, E., Moskaluk, C.A., Bickston, S.J., Cominelli, F., 1999. IL-18, a novel immunoregulatory cytokine, is up-regulated in Crohn's disease: Expression and localization in intestinal mucosal cells. J Immunol 162, 6829-6835. 
Rallabhandi, P., Awomoyi, A., Thomas, K.E., Phalipon, A., Fujimoto, Y., Fukase, K., Kusumoto, S., Qureshi, N., Sztein, M.B., Vogel, S.N., 2008. Differential activation of human TLR4 by Escherichia coli and Shigella flexneri 2a lipopolysaccharide: Combined effects of lipid A acylation state and TLR4 polymorphisms on signaling. J Immunol 180, 1139-1147.

Roses, R.E., Xu, S.W., Xu, M., Koldovsky, U., Koski, G., Czerniecki, B.J., 2008. Differential production of IL-23 and IL-12 by myeloid-derived dendritic cells in response to TLR agonists. J Immunol 181, 51205127.

Sakuraba, A., Sato, T., Kamada, N., Kitazume, M., Sugita, A., Hibi, T., 2009. Th1/Th17 Immune Response Is Induced by Mesenteric Lymph Node Dendritic Cells in Crohn's Disease. Gastroenterology $137,1736-1745$.

Sivakumar, P.V., Westrich, G.M., Kanaly, S., Garka, K., Born, T.L., Derry, J.M.J., Viney, J.L., 2002. Interleukin 18 is a primary mediator of the inflammation associated with dextran sulphate sodium induced colitis: blocking interleukin 18 attenuates intestinal damage. Gut 50, 812-820.

Sokol, H., Lepage, P., Seksik, P., Dore, J., Marteau, P., 2006. Temperature gradient gel electrophoresis of fecal 16S rRNA reveals active Escherichia coli in the microbiota of patients with ulcerative colitis. J Clin Microbiol 44, 3172-3177.

Sondergaard, J.N., Brix, S., 2012. Isolation of IL-12p70-competent human monocyte-derived dendritic cells. J immunol Methods 386, 112-116.

Soong, G., Chun, J., Parker, D., Prince, A., 2012. Staphylococcus aureus Activation of Caspase 1/Calpain Signaling Mediates Invasion Through Human Keratinocytes. J Infect Dis 205, 1571-1579.

Vejborg, R.M., Hancock V., Petersen A.M., Krogfelt K.A., Klemm P. 2011. Comparative genomics of Escherichia coli isolated from patients with inflammatory bowel disease. BMC Genomics 12, 316-328. 


\section{Figure legends}

Figure 1. Response profile in dendritic cells upon challenge with viable $E$. coli strains. A, A, Comparison of cytokine responses from monocyte-derived dendritic cells (moDCs) exposed to lipopolysaccharide (LPS) (1 $\mathrm{gg} / \mathrm{mL})$ and LF82 at MOI 1, 5 and 10 in supernatants harvested upon $20 \mathrm{~h}$ of stimulation. At $1 \mathrm{~h}$ post challenge, $50 \mu \mathrm{g} / \mathrm{mL}$ gentamicin was added to all moDC cultures to kill extracellular bacteria. Data show the response from one representative of 11 individuals and display the median of technical replicates $(n=3)$ with bars representing the $\mathrm{min} / \mathrm{max}$ values. $\mathbf{B}$, Cytokine profiles in supernatants from moDCs exposed to LF82, ECN and C691-04A (Table 1) at MOI 1, 5 and 10 for $20 \mathrm{~h}$, and otherwise treated as in A. Cytokine levels are show as the median of 11 donors displayed as $\mathrm{pg} / \mathrm{mL}$ on a log scale. C, Comparison of IL-18 levels from moDCs after $20 \mathrm{~h}$ of stimulation with LF82, ECN, C691-04A and C311-05 at MOI 1 where each dot represents moDCs from one donor. KruskalWallis test followed by Dunn's Multiple Comparison Test was used for statistical comparison. ${ }^{* * *}, p \leq$ $0.001{ }^{* *}, p \leq 0.01 ; \mathrm{ns}=$ non-significant. $\mathbf{D}$, The cytokine signature from moDCs exposed to LF82 and C691-04A at MOI 1, 5 and 10. Data show the percentage of the individual cytokines in relation to their total amount, with original data shown in B ( $n=11$ blood donors). LF82 data are representative for all examined E. coli strains, except for C691-04A and C311-05. E, Total abundance plots of the measured cytokines from moDCs for LF82 and C691-04A at MOI 1, 5 and 10 ( $n=11$ blood donors).

Figure 2. Dendritic cell viability after exposure to viable $E$. coli strains. A, Viability plots of mature moDCs exposed to $20 \mathrm{~h}$ of stimulation with medium, LPS $(1 \mu \mathrm{g} / \mathrm{mL})$ and LF82 at MOI 1, 5 and 10 . Cells were stained with the dead-marker stain, SYTOX ${ }^{\circledR}$ AADvanced (AAD) and subjected to flow cytometric analysis. The plot displays AAD versus side scatter (SSC) of 120,000 moDCs. The encircled population 
make up the viable population (AAD-negative, high SSC). The other AAD-negative population with low SSC represents apoptotic cells, while the AAD-positive cells make up necrotic cells. Data are representative for 5 donors. B, Viability of moDCs after $20 \mathrm{~h}$ of stimulation with the $13 \mathrm{E}$. coli isolates, determined as the percentage of SYTOX ${ }^{\circledR}$ AADvanced (AAD)-negative and SSC-high cells after flow cytometry (encircled population in A). The spider-plot represents the median viability of bacteriatreated moDCs from 5 blood donors.

Figure 3. Divergent viability dynamics in E. coli-exposed dendritic cells. A, SYTOX ${ }^{\circledR}$ AADvanced (AAD) versus side-scatter (SSC) biplots showing five different dendritic cell (moDC) subsets $(V, D 1, D 2, D 3$ and $\mathrm{D4}$ ) promoted by LF82 at $\mathrm{MOI} 1,5$ and 10 after $4 \mathrm{~h}$ of stimulation, and visualized by flow cytometry. Cultures were added $50 \mu \mathrm{g} / \mathrm{mL}$ gentamicin at $1 \mathrm{~h}$ post challenge. B, Forward scatter (FSC) versus SSC overlay biplots from the analysis in A, exposing the FSC-SSC properties of the individual subpopulations ( $V, D 1, D 2, D 3$ and $D 4)$ emerging upon $4 \mathrm{~h}$ challenge with LF82 at different MOls. C, AAD-staining vs. SSC in moDCs exposed to E. coli Nissle (ECN), C691-04A and C3111-05 for $4 \mathrm{~h}$ at MOI 1, 5 and 10. D, Biplots displaying the FSC-SSC properties of the moDC subtypes from C. All data (A-D) are representative for 5 blood donors.

Figure 4. Some $E$. coli spp. induce distinct dendritic cell death modes during early stages of infection. A, Comparison of C691-04A- and LF82-induced dendritic cell (moDC) subtypes ( $V, D 1, D 2$, $D 3$ and $D 4$ ) at 4 h post challenge with $M O I 5$, displayed as SYTOX ${ }^{\circledR}$ AADvanced (AAD) versus side 
scatter (SSC) (left biplots). The viable ( $V$ ) population is further divided into two populations ( $D 5$ and VO) based on forward-scatter (FSC) properties (A, right biplots). D5 represents a FSC-low phenotype that stains AAD-negative. B, Comparison of the distribution of the $V$ subpopulation into $D 5$ and $V 0$, as induced by different MOls of C691-04A at $4 \mathrm{~h}$ and $20 \mathrm{~h}$ post challenge. All data $(A, B)$ are representative for 5 blood donors.

Figure 5. Similar induction of ROS production in moDC across the $E$. coli spp. A, Production of intracellular reactive oxygen species (ROS) in the six moDC subpopulations (VO, D1, D2, D3, D4 and D5) mediated by C691-04A after 4 and $20 \mathrm{~h}$ of stimulation. Cells, processed as in Fig. 4B, were costained with DCFH-DA, and subjected to flow cytometric analysis to determine ROS production. The mean fluorescence intensity (MFI) of the six moDC subpopulations represents the ROS amount produced within the individual moDC populations. Data are representative for 5 donors. B, ROS production in viable moDCs (=VO/V) treated with viable LF82, ECN, C691-04A or C311-05 at different MOIs relative to that in medium-treated moDCs. Data are medians of the individualized normalized ratios from 5 blood donors with bars indicating min and max values.

Figure 6. E. coli C691-04A and E. coli Nissle show identical cytopathic effect on intestinal epithelium cells at very high MOI. A, B, IL-8 and IL-18 secretion from intestinal epithelial cells (IECs) exposed to viable LF82, E. coli Nissle (ECN) and C691-04A at MOI 100, 500 and 1000 for 20 h. C, IEC viability $20 \mathrm{~h}$ post challenge with the indicated viable bacteria at different MOls. D, Comparison of intracellular 
reactive oxygen species (ROS) production induced by the three viable $E$. coli strains $20 \mathrm{~h}$ post challenge. A-D, IECs were exposed to viable $E$. coli for $1 \mathrm{~h}$, followed by addition of $50-100 \mu \mathrm{g} / \mathrm{ml}$ gentamicin (depending on $\mathrm{MOI}$ ) for the remaining $17 \mathrm{~h}$ of stimulation. Data show medians with bars indicating min and max values based on three individual experiments. 